\title{
iMed. New procedure for the synthesis of indolo[3,2-b]quinoline ULisboa derivatives with DNA G-quadruplex stabilization capacity
}

Adriana M. D. Silva ${ }^{1}$, Eduarda Mendes ${ }^{1}$, Maria M. Santos ${ }^{1}$, Alexandra Paulo ${ }^{1}$

${ }^{1}$ Research Institute for Medicines, Faculty of Pharmacy, Universidade de Lisboa, Portugal

Email: adrianamsilva@campus.ul.pt, mapaulo@ff.ulisboa.pt

\section{Introduction}

Cancer is one of the leading causes of death worldwide and the occurrence of resistance to common anticancer drugs demands new and innovative drug design approaches. G-quadruplexes (G4s) DNA structures in oncogenic promoter regions (of $c-M Y C$ and KRAS oncogenes, for example) and telomeres are potential targets for cancer therapy. Small molecules could serve as DNA G4 stabilizers and down-regulate the targeted gene expression leading to induction of programmed cell death by apoptosis [1][2]

Indolo[3,2-b]quinoline and indolo[3,2-c]quinoline derivatives were previously reported as stabilizers of G4 DNA structures (Figure 1) and promising selective anticancer leads, as these compounds are able to preferentially target the G4 motifs in the KRAS promoter and inhibit the transcription and translation of this oncogene, inducing apoptosis of KRAS-dependent colon cancer cells by up-regulating the expression of the pro-apoptotic transcription factor p53 (Figure 2) [3][4]. Activation of p53 by small molecules is also expected to be a valuable approach in fighting cancer. In this area of research, we have previously identified other promising indole-based compounds with activity as p53 gene expression or p53 function activators [5][6]. Thus, the improvement of the synthetic procedures of these compounds are highly relevant. Herein we describe an alternative experimental procedure for the synthesis of 7-carboxylate indolo[3,2-b]quinoline tri-alkylamine derivatives 1a and 2a.

A

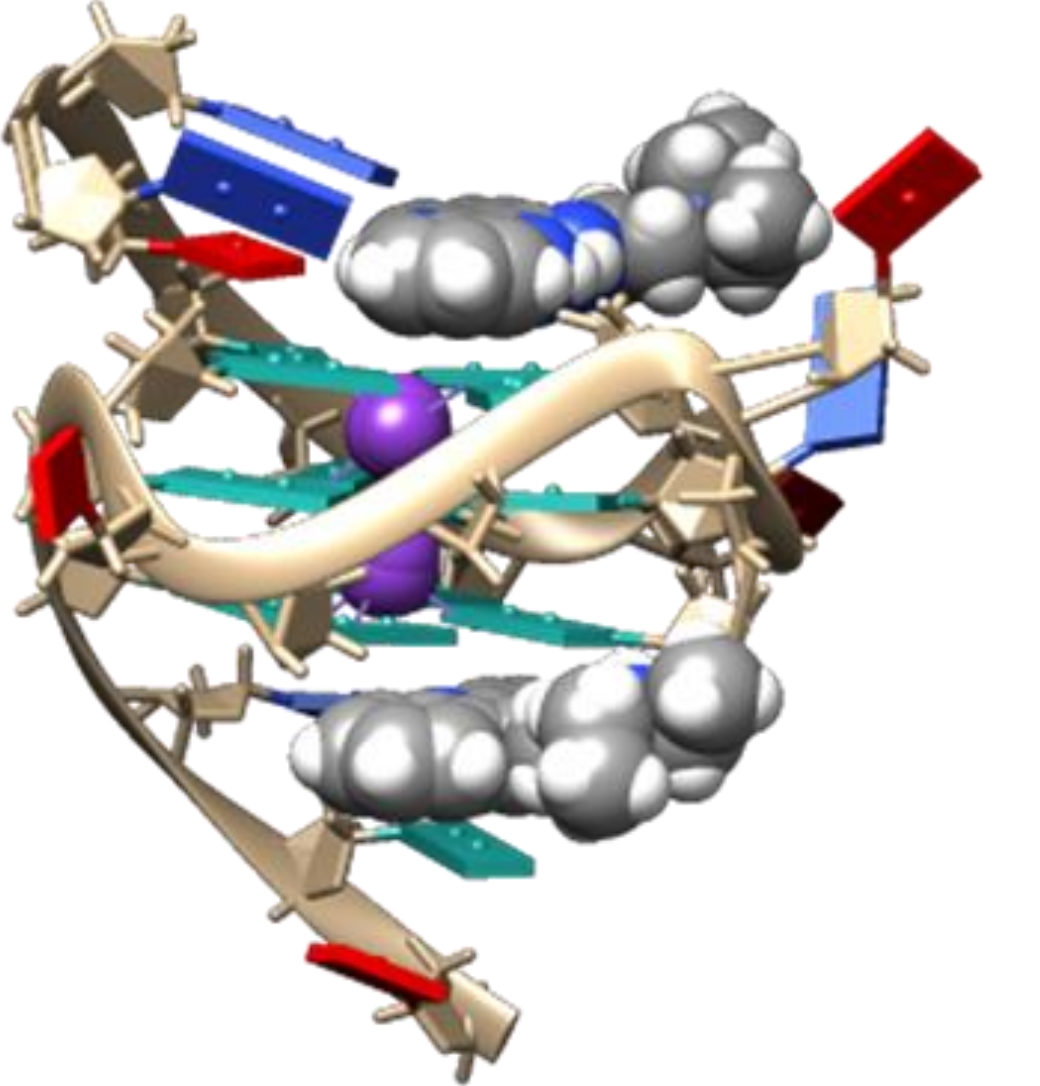

B

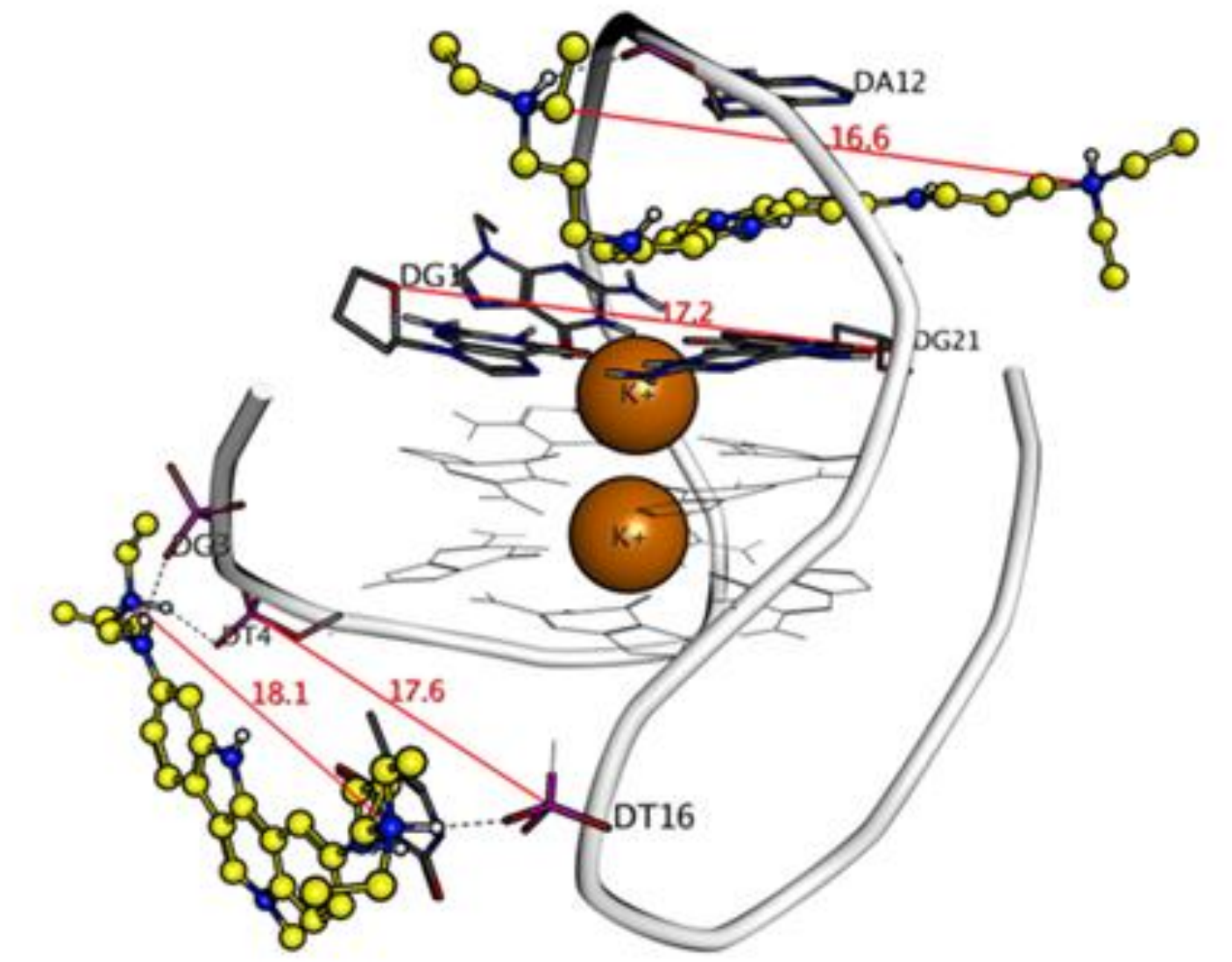

Figure 1-A) Structure of 2:1 complex of an indolor3 2-blquinoline derivative with c-MYC promoter G4 (PDB ID: (17V). B) Molecular of 2.1 complex of an indolo [3,2-b]quinoline derivative with C-MYC promoter G4 (PDB ID: telemore G4 (PDB ID: 143D).[7]

\section{Synthetic Procedures}<smiles>Nc1ccccc1C(=O)OCCCCCC(=O)O</smiles><smiles>CCOC(=O)c1ccc(NC(=O)COc2ccc(CC(C)C)cc2)cc1</smiles>

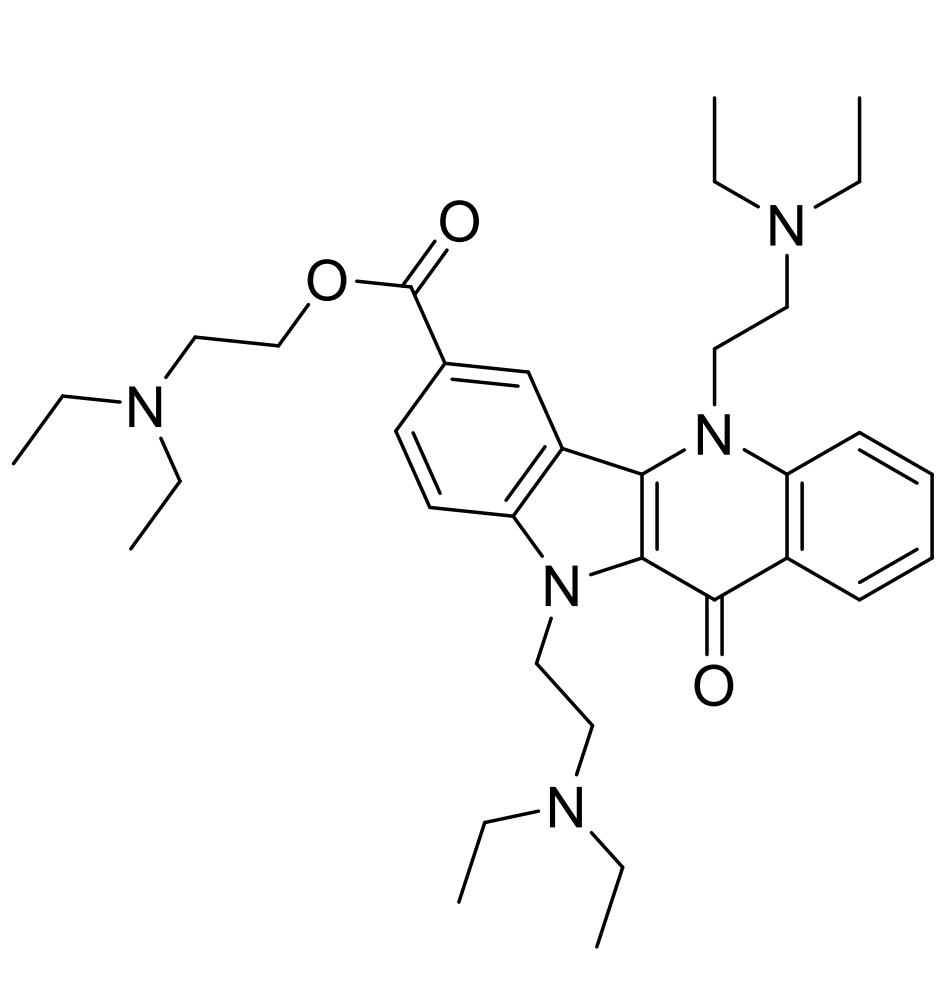

Compounds $\mathbf{1 a}$ and $\mathbf{2 a}$ were synthesized as previously described [3], with the following modifications: -Synthesis of intermediate 5 :

\section{A mixture of compound 4 and 4}

ethylaminobenzoate in DMF was placed in a

microwave apparatus, at $300 \mathrm{~W}, 140^{\circ} \mathrm{C}$, for 4

hours. Yield: $62 \%$.

A mixture of compound 4 and 4 -

ethylaminobenzoate in DMF was heated at

$140^{\circ} \mathrm{C}$ in a pressure tube for 6 hours. Yield: $40-$ $60 \%$.

-Work-up procedure for intermediate 6 :

\section{The reaction mixture is basified to $\mathrm{pH} 4$ with} $\mathrm{KOH}$ and the product is isolated by liquid-liquid extraction with ethyl acetate. Yield: $20 \%$.

The reaction mixture is basified to $\mathrm{pH} 4$ with $\mathrm{KOH}$ and the dark green sticky precipitate that is formed is filtered and then dissolved in metanol. The solubilized product is separated from unsoluble by-products by filtration. Yield: $66 \%$.

G-quadruplex stabilization by FRET melting assay

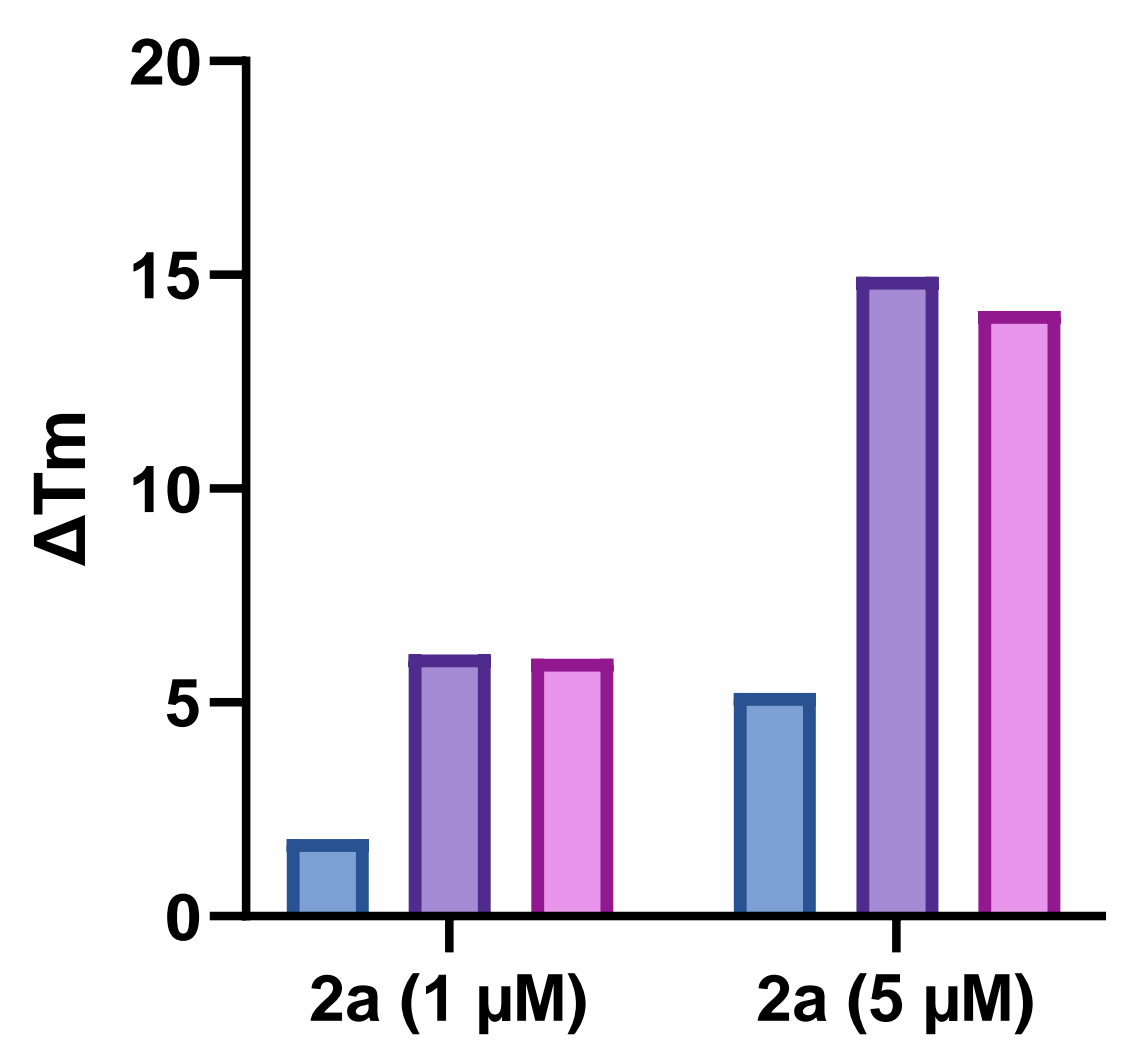

$$
\begin{aligned}
& \text { ㅁ-loop } \\
& \text { ㅁ F21T } \\
& \text { KRAS }
\end{aligned}
$$

Indolo[3,2-b]quinoline 2a preferentially binds and stabilize DNA G4 than ds-DNA. Melting temperature variations $(\triangle T \mathrm{Tm})$ of labeled $\mathrm{G} 4 \mathrm{~s}$ present in promoters of $k$-RAS (KRAS), human telomere (F21T) and hairpin loop sequence (Tloop) at $0.2 \mu \mathrm{M}$, stabilized by compound $2 \mathrm{a}$. $\Delta \mathrm{Tm}$ values are averages from a triplicate experiment std errors $<0.25^{\circ} \mathrm{C}$.

\section{Conclusions}

With the herein reported alternative method for the synthesis of intermediate $\mathbf{5}$ the microwave apparatus can be replaced by a pressure tube, a much more economic lab equipment.

An improved work-up procedure to obtain intermediate 6 is also here reported. With this procedure the yield increases from $20 \%$ to $66 \%$

Spectra

a)

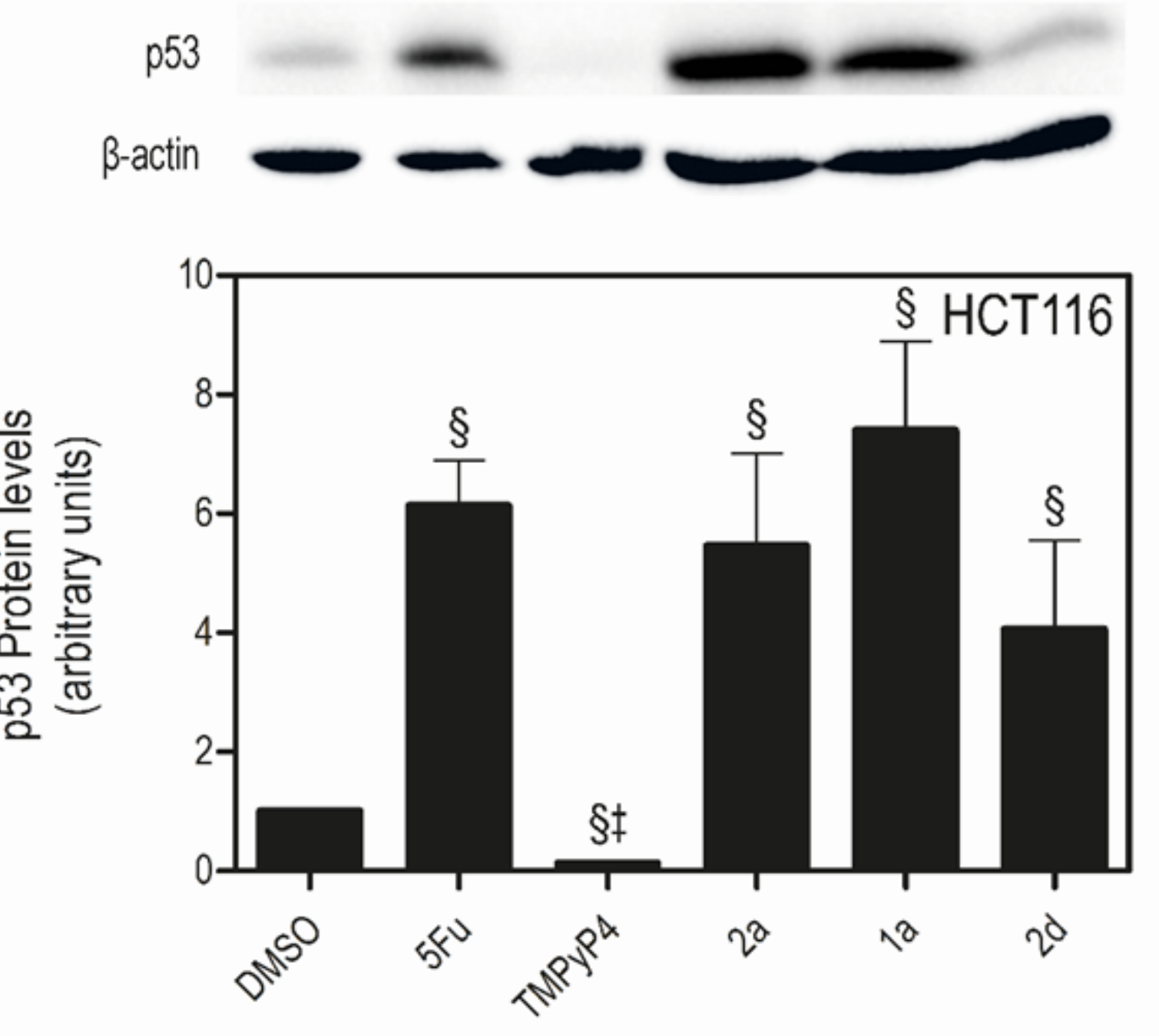

Figure 2 - Exposure to indolo[3,2b]quinoline derivatives $1 \mathrm{a}$ and $2 \mathrm{a}$ increases p53 steady-state protein from [3])

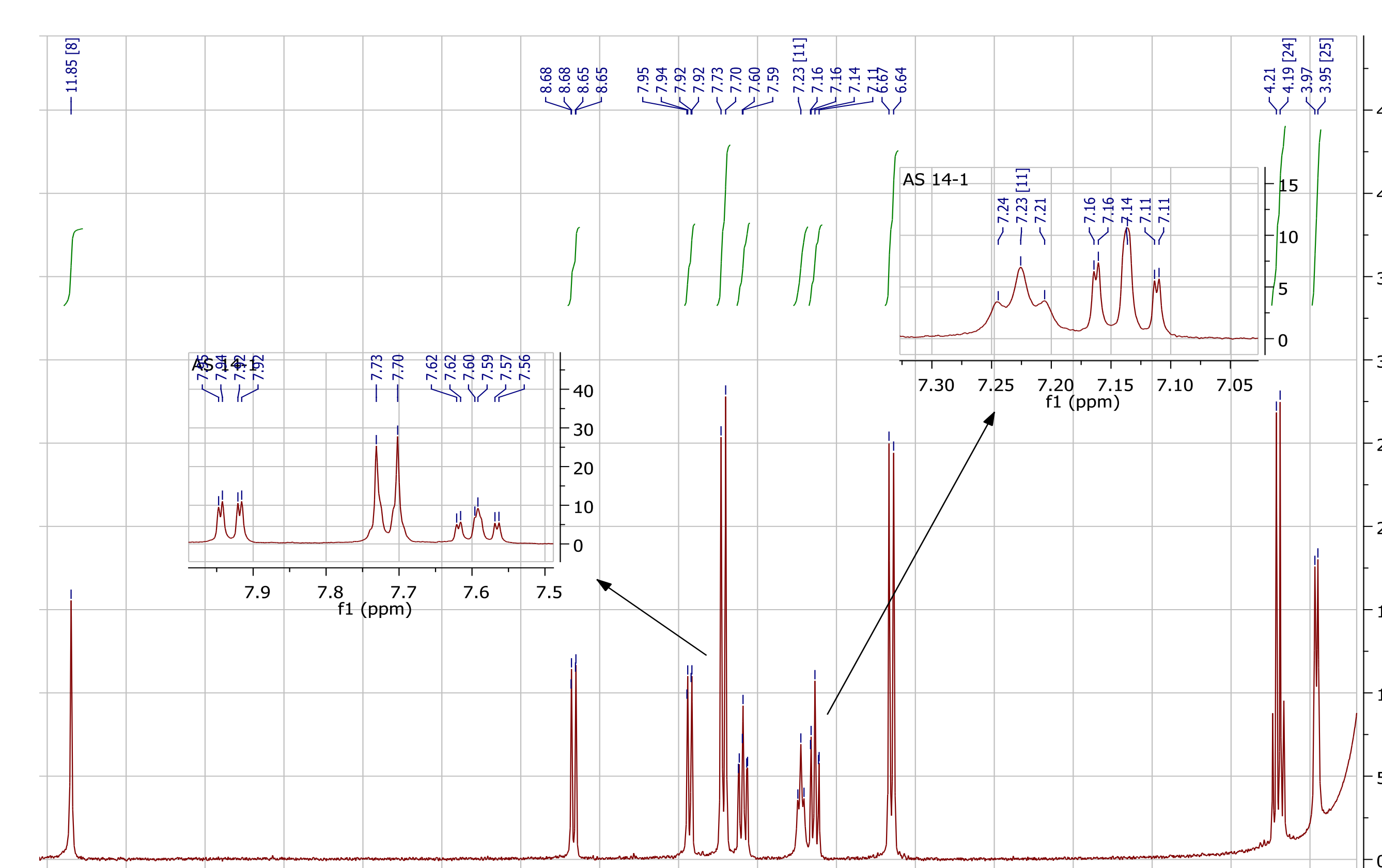

b)

c)

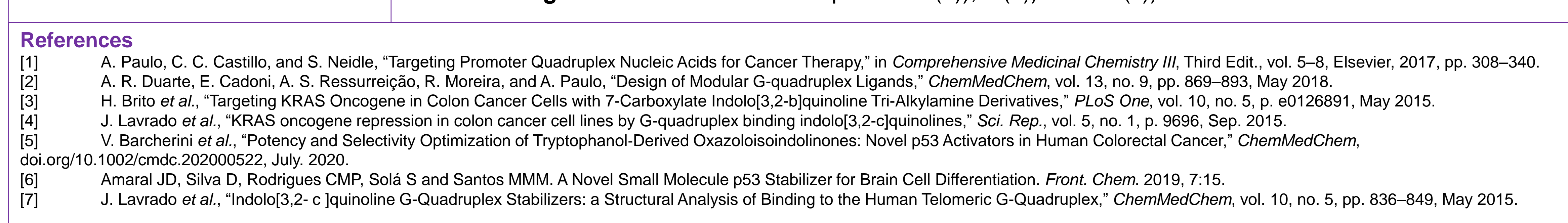

This work was supported by National Funds (Fundação para a Ciência e Tecnologia) through iMed.ULisboa (UIDB/04138/2020) and project PTDC/QUI-QOR/29664/2017 